Gut and Liver, Vol. 14, No. 6, November 2020, pp. 817-825

\title{
Endoscopic Ultrasound-Guided Tissue Acquisition by 22-Gauge Franseen and Standard Needles for Solid Pancreatic Lesions
}

Kazunaga Ishigaki ${ }^{1}$, Yousuke Nakai ${ }^{1,2}$, Hiroki Oyama ${ }^{1}$, Sachiko Kanai ${ }^{1}$, Tatsunori Suzuki ${ }^{1}$, Tomoka Nakamura ${ }^{1}$, Tatsuya Sato ${ }^{1}$, Ryunosuke Hakuta ${ }^{1}$, Kei Saito $^{1}$, Tomotaka Saito ${ }^{1}$, Naminatsu Takahara ${ }^{1}$, Tsuyoshi Hamada ${ }^{1}$, Suguru Mizuno ${ }^{1}$, Hirofumi Kogure ${ }^{1}$, Minoru Tada ${ }^{1}$, Hiroyuki Isayama ${ }^{3}$, and Kazuhiko Koike ${ }^{1}$

Departments of ${ }^{1}$ Gastroenterology and ${ }^{2}$ Endoscopy and Endoscopic Surgery, Graduate School of Medicine, The University of Tokyo, and ${ }^{3}$ Department of Gastroenterology, Graduate School of Medicine, Juntendo University, Tokyo, Japan

Background/Aims: Recently, a three-plane symmetric needle with Franseen geometry was developed for endoscopic ultrasound-guided fine needle biopsy (EUS-FNB). In this retrospective study, tissue acquisition per pass was compared between 22-gauge Franseen FNB and standard fine needle aspiration (FNA) needles in patients with solid pancreatic lesions. Methods: Consecutive patients who underwent EUSFNA or EUS-FNB for solid pancreatic lesions between October 2014 and March 2018 were retrospectively studied. The tissue acquisition rate and the diagnostic performance per session, per pass, and at first pass were compared. Results: A total of 663 passes (300 by the FNB needle and 363 by the standard FNA needle) were performed in 154 patients (71 FNB and 83 FNA). The tissue acquisition rate per session and at first pass in the FNB and FNA groups was 100\% and $95 \%(p=0.13)$ and $87 \%$ and $69 \%(p=0.007)$, respectively. The multivariate analysis revealed that among the patients, EUS-FNB (odds ratio, 3.07; $p=0.01$ ) was associated with a higher first-pass tissue acquisition rate. While the tissue acquisition rate reached a plateau after the 4th pass with FNA, it reached a plateau after the 2 nd pass with FNB. Among the 129 malignant cases, the histological tissue acquisition rate per session was similar (100\% and $94 \%$ ), but the sensitivity by histology alone per session was higher for FNB than for FNA (93\% and 73\%, p<0.01). Conclusions: The results of our retrospective analysis indicated that compared with a standard FNA needle, a 22-gauge Franseen FNB needle was associated with a higher first-pass tissue acquisition rate. (Gut Liver 2020;14:817-825)

Key Words: Endoscopic ultrasound; Endoscopic ultrasound- guided fine needle aspiration; Endoscopic ultrasonographyguided fine-needle biopsy; Histology; Pancreatic neoplasms

\section{INTRODUCTION}

Endoscopic ultrasound-guided fine needle aspiration (EUSFNA) is an established procedure for tissue acquisition and pathological diagnosis of pancreatic solid lesions. ${ }^{1-3}$ However, histological tissue acquisition of EUS-FNA is not necessarily sufficient for additional diagnostic techniques or for the identification of molecular markers often necessary for the final diagnosis and appropriate clinical management. To overcome these disadvantages of EUS-FNA, several novel needles have been introduced for EUS-guided fine needle biopsy (EUS-FNB). ${ }^{4-20}$

Recently, a three-plane symmetric needle with Franseen geometry has been developed for performing EUS-FNB. ${ }^{15-20}$ While rapid on-site evaluation (ROSE) reportedly increases the diagnostic performance and decreases the number of passes, ${ }^{21}$ its role in EUS-FNB is not fully established in clinical practice. In addition, ROSE is not readily available in many institutions including our center. We conducted this retrospective study comparing tissue acquisition rates per pass between 22-gauge Franseen FNB needle and 22-gauge standard FNA needle to evaluate the role of EUS-FNB without ROSE in patients with pancreatic solid lesions.

\section{MATERIALS AND METHODS}

\section{Patients}

This is a single center, retrospective study in the University of Tokyo Hospital. Data on consecutive patients who underwent

Correspondence to: Yousuke Nakai

Department of Endoscopy and Endoscopic Surgery, Graduate School of Medicine, The University of Tokyo, 7-3-1 Hongo Bunkyo-ku, Tokyo 1138655, Japan

Tel: +81-3-3815-5411, Fax: +81-3-5800-9801, E-mail: ynakai-tky@umin.ac.jp

Received on May 16, 2019. Revised on August 12, 2019. Accepted on August 13, 2019. Published online May $29,2020$.

pISSN 1976-2283 eISSN 2005-1212 https://doi.org/10.5009/gnl19171

@ This is an Open Access article distributed under the terms of the Creative Commons Attribution Non-Commercial License (http://creativecommons.org/licenses/by-nc/4.0) which permits unrestricted non-commercial use, distribution, and reproduction in any medium, provided the original work is properly cited. 
EUS-FNA between October 2014 and September 2016 and EUSFNB between October 2016 and March 2018 for pancreatic solid lesions were retrieved from a prospectively maintained database at the University of Tokyo Hospital. Inclusion criteria were patients who underwent EUS-guided tissue acquisition using a standard 22-gauge FNA needle (Expect needle: Boston Scientific Japan, Tokyo, Japan) or a 22-gauge EUS-FNB needle (Acquire needle: Boston Scientific Japan) for pancreatic solid lesions and whose cytological and histological analyses per passes were available. Exclusion criteria were patients with severe coagulopathy and patients with a pancreatic cystic lesion or an extrapancreatic lesion. This study was approved by the local ethical committee (approval number: 1804) and all patients provided informed consent for EUS-guided tissue acquisition.

\section{EUS-FNA or EUS-FNB procedure}

All EUS procedures were performed using a curved linear array echoendoscope (GF-UCT260; Olympus Medical Systems, Tokyo, Japan or EG-580-UT; Fujifilm Medical Systems, Tokyo, Japan), which was connected to a processor featuring color Doppler function (EU-ME2; Olympus Medical Systems or SU-1, Fujifilm Medical Systems) under moderate sedation with intravenous midazolam and pethidine hydrochloride. After EUS evaluation including regional vasculature with color Doppler function, EUS-guided tissue acquisition was performed. The FNA or FNB needle with a stylet was advanced into the target lesion under EUS guidance. A stylet was completely removed and 10 to 20 to-and-fro movements within the target lesion were performed while a 10-mL suction was applied. The needle was withdrawn after suction was released, and the obtained material was expressed entirely onto a glass slide by reinsertion of the stylet. The material on the slide was then carefully inspected and visible core, if any, was lifted off the slide and placed into a formalin bottle for histologic examination. The remaining specimen was sent for cytological examination. No ROSE was performed. The number of passes was decided at the discretion of the attending physician but EUS-FNA or FNB was basically repeated until enough visible core tissue was obtained macroscopically (Fig. 1). Cytological and histological reports were retrospectively reviewed. Cellularity and blood contamination for each slide of cytological specimen were evaluated based on the consensus between two cytotechnologists who were blind to FNA or FNB needles, and the semi-quantified scores used in this study were routinely recorded in the pathological reports as follows; 0 (none), 1 (few aggregates), 2 (fair cellularity) and 3 (abundant cellularity) for cellularity and 0 (none), 1 (few), 2 (moderate) and 3 (high) for blood contamination. Cellularity score was based on the amount of all types of cells including tumor cells. Pathological diagnosis was based on the combination of cytological and histological diagnoses as previously reported. ${ }^{22}$ All procedures were performed by 10 experts ( $\geq 5$ years of EUS-FNA experiences) or by 12 trainees ( $<5$ years of EUS-FNA experiences) under

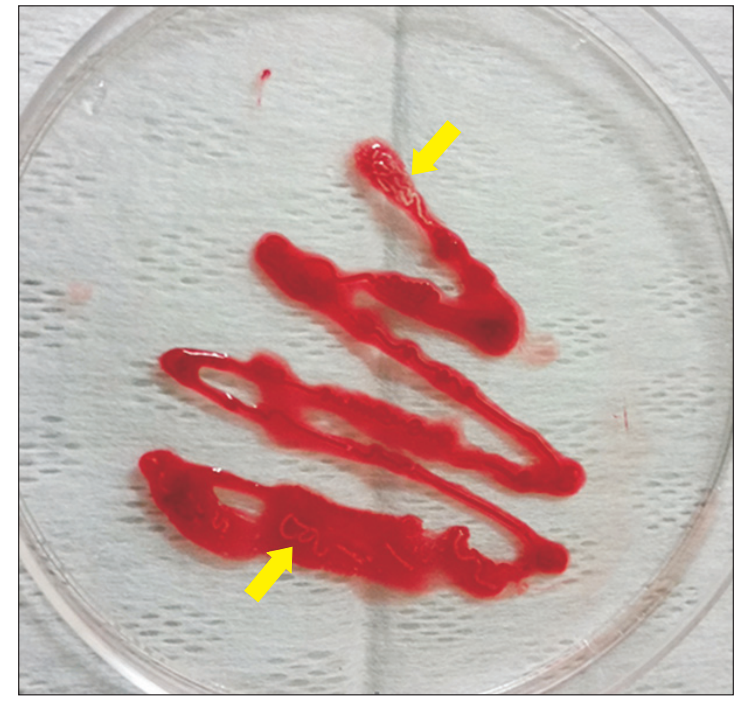

Fig. 1. Macroscopic findings of endoscopic ultrasound-guided fine needle biopsy specimens. A whitish core tissue was clearly observed upon visual inspection (arrows).

supervision by experts.

\section{Final diagnosis}

The final diagnosis was made either by the pathological diagnosis based on surgically resected specimen, by EUS-FNA or EUS-FNB diagnosis positive for malignancy with compatible clinical outcomes, or by EUS-FNA or EUS-FNB diagnosis negative for malignancy with lack of deterioration on follow-up more than 6 months.

\section{Outcome measurements}

Primary endpoint of this study was the histological tissue acquisition rate in a first pass and per session. The histological tissue acquisition rate was defined as the acquisition rate of the sufficient material for adequate histological interpretation. Secondary outcomes were the diagnostic performance by histology, cytology and combination per pass, per session and in the first pass, cellularity and bloodiness of cytological specimen per pass, adverse events and the prognostic factors for first-pass tissue acquisition and histological sensitivity. Technical failure was defined as failure to obtain macroscopic visible specimen before needle exchange. Adverse events were defined and graded according to the lexicon. ${ }^{23}$ Post EUS-FNA or FNB pancreatitis was defined as a new onset of abdominal pain or a worsening of an existing one, associated with an increase in amylase or lipase more than 3 times the upper cutoff value. Computed tomography was performed for evaluation of pancreatitis in those cases with abdominal pain associated with elevated pancreatic enzymes. 


\section{Statistical analysis}

Continuous variables were presented as the median and range, and categorical variables as the number and percentage. Statistical comparisons were performed with the chi-square test or the Fisher exact test for categorical variables and the Wilcoxon rank-sum test for continuous variables. Logistic regression models were used to evaluate prognostic factors of first-pass tissue acquisition and histological sensitivity. Potential prognostic factors of first-pass tissue acquisition were age, sex, tumor size, tumor location, puncture route, needle type, and final diagnosis of pancreatic cancer. A p-value of $<0.05$ in a two-tailed test was considered as a statistically significant difference. All statistical analyses were performed with EZR (Saitama Medical Center, Jichi Medical University, Saitama, Japan). ${ }^{24}$

\section{RESULTS}

\section{Patient characteristics and EUS procedures}

A total of 663 passes ( 300 by the FNB needle and 363 by the FNA needle) were performed in 154 patients (71 FNB and 83 FNA) with pancreatic solid lesions during the study period. Patient characteristics and final diagnoses are shown in Table 1. In both groups, the final diagnosis was pancreatic cancer in $72 \%$. The median tumor size was $25 \mathrm{~mm}$ in the FNB group and 24 $\mathrm{mm}$ in the FNA group. The median number of passes was four in both groups.

In the FNA group, there was one technical failure case in which macroscopic visible specimen could not be obtained before needle exchange to a 25-gauge FNA needle. In additional 14 cases (four EUS-FNB and 10 EUS-FNA), though EUS-FNB or EUS-FNA was technically successful, the needle was exchanged to a 25-gague FNA needle at the discretion of endoscopists. The

Table 1. Patient Characteristics and EUS-Guided Tissue Acquisition

\begin{tabular}{|c|c|c|c|}
\hline Characteristics & FNB $(n=71)$ & FNA $(\mathrm{n}=83)$ & $\mathrm{p}$-value \\
\hline Age, yr & $68(26-85)$ & $70(32-93)$ & 0.65 \\
\hline Male sex & $46(65)$ & $49(59)$ & 0.51 \\
\hline \multicolumn{4}{|l|}{ Final diagnosis } \\
\hline Malignant & $57(80)$ & $72(87)$ & \\
\hline Pancreatic cancer & $51(72)$ & $60(72)$ & \\
\hline Neuroendocrine tumor & $3(4)$ & $8(10)$ & \\
\hline Metastatic pancreatic tumor & $3(4)$ & $4(5)$ & \\
\hline Benign & $14(20)$ & $11(13)$ & \\
\hline Autoimmune pancreatitis & $5(7)$ & $5(6)$ & \\
\hline Chronic pancreatitis & $3(4)$ & $2(2)$ & \\
\hline Nonspecific inflammation & $3(4)$ & $2(2)$ & \\
\hline Serous cyst neoplasm & $1(1)$ & $1(1)$ & \\
\hline Pancreatic abscess & $1(1)$ & $1(1)$ & \\
\hline Schwannoma & $1(1)$ & 0 & \\
\hline Tumor size, mm & $25(5-66)$ & $24(7-67)$ & 0.65 \\
\hline Tumor location & & & 0.90 \\
\hline Head & $29(41)$ & $36(43)$ & \\
\hline Body & $31(44)$ & $36(43)$ & \\
\hline Tail & $11(15)$ & $11(13)$ & \\
\hline Puncture site & & & 0.50 \\
\hline Stomach & $39(55)$ & $51(61)$ & \\
\hline Bulb & $25(35)$ & $22(27)$ & \\
\hline D2 & $7(10)$ & $10(12)$ & \\
\hline No. of passes & $4(2-8)$ & $4(1-8)$ & 0.29 \\
\hline Procedures & & & 0.01 \\
\hline Experts & $32(45)$ & $56(67)$ & \\
\hline Trainee & $39(55)$ & $27(33)$ & \\
\hline
\end{tabular}

Data are presented as median (range) or number (\%).

EUS, endoscopic ultrasound; FNB, fine needle biopsy; FNA, fine needle aspiration; D2, the second portion of the duodenum. 

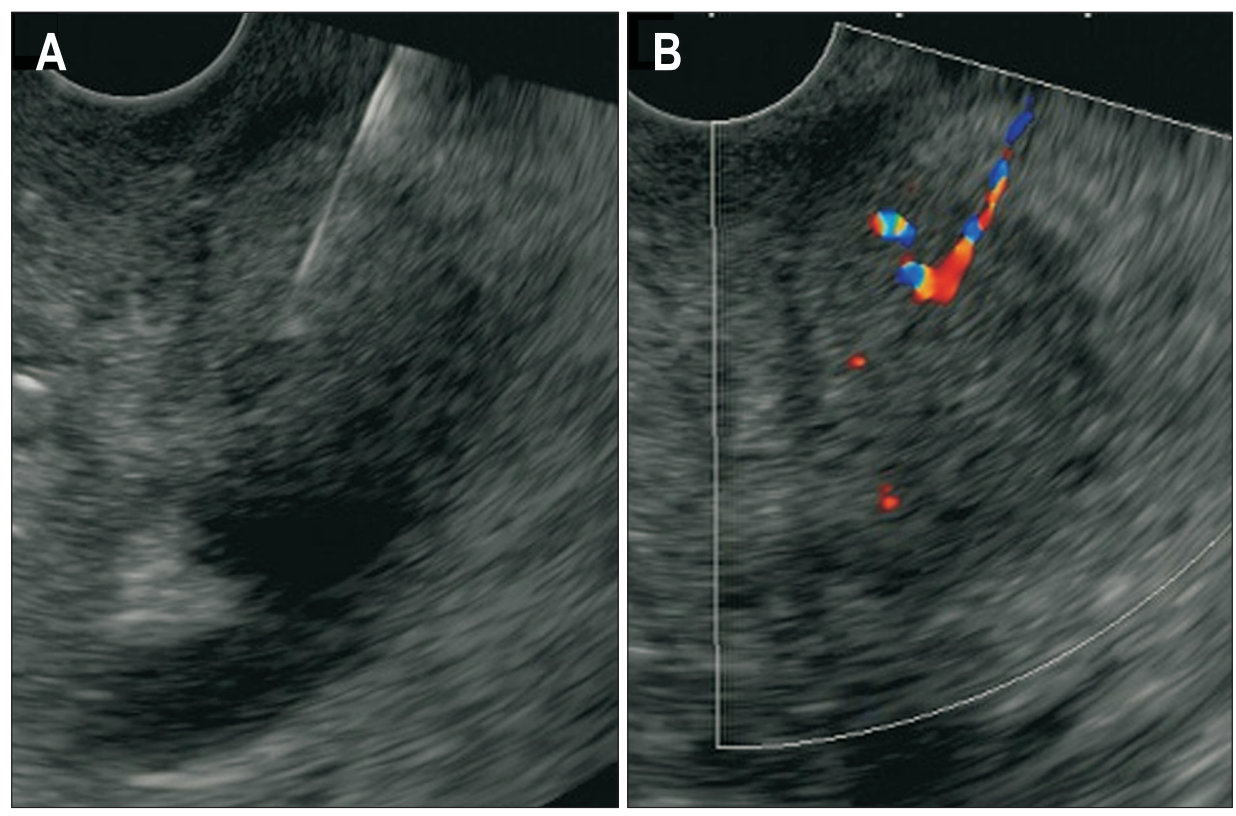

Fig. 2. (A) The EUS image of EUSFNB puncture. (B) After removal of the FNB needle, a Doppler signal was temporarily observed along the needle tract.

EUS, endoscopic ultrasound; FNB, fine needle biopsy.

reasons for needle exchange were difficult angle for puncture in 10 (three EUS-FNB and seven EUS-FNA) and needle malfunction in four (one EUS-FNB and three EUS-FNA).

As procedure-related adverse events, two cases (4\%) in the FNB group developed mild pancreatitis. Doppler signal along the needle tract was observed in some cases after EUS-FNB (Fig. 2) but clinically overt bleeding was not observed. Doppler signal along the needle tract was rarely observed in the FNA group. There were no adverse events in the FNA group.

\section{Histological tissue acquisition in the FNB and FNA groups}

The histological tissue acquisition rate in the first pass was significantly higher in the FNB group: 87\% and 69\% in the FNB and FNA groups $(\mathrm{p}=0.007)$, respectively. After a median of four passes in both groups, the histological tissue acquisition rate was 100\% and 95\% in the FNB and FNA groups ( $p=0.13$ ), respectively. While tissue acquisition rate reached a plateau after 4th pass with FNA, it reached a plateau after 2nd pass with FNB (Fig. 3). The histological tissue acquisition was confirmed after the procedure, so the median number of passes was same in both groups.

Prognostic factor analyses of first-pass tissue acquisition are shown in Table 2. In the multivariate analysis, EUS-FNB (odds ratio [OR], 3.07; 95\% confidence interval, 1.31 to $7.17 ; \mathrm{p}=0.01$ ) and puncture from the stomach $(0 R, 0.42 ; 95 \%$ confidence interval, 0.18 to $0.98, \mathrm{p}=0.04$ ) were the significant factors associated with a higher tissue acquisition rate.

\section{Cytological evaluation in the FNB and FNA groups}

Results of cytological evaluation are shown in Table 3. The accuracy, sensitivity, specificity, positive and negative predictive

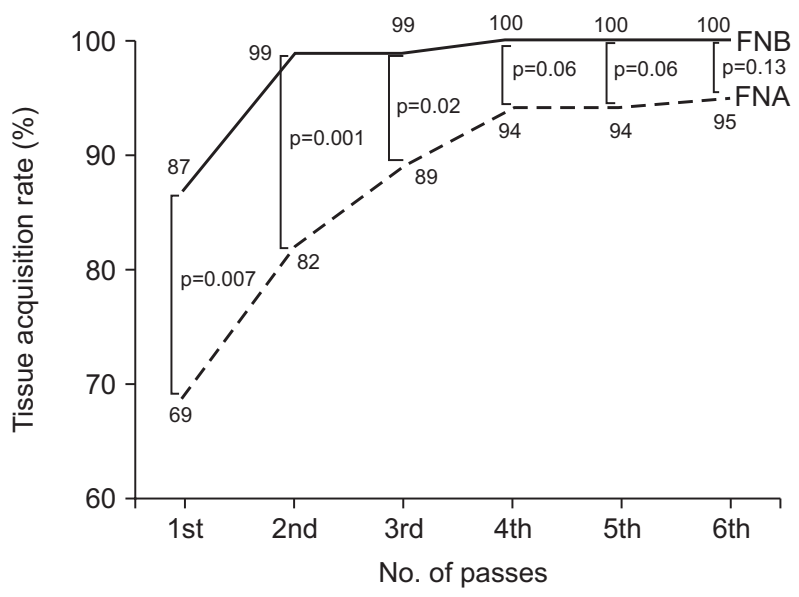

Fig. 3. Tissue acquisition rates of EUS-FNB and EUS-FNA. The tissue acquisition rate reached a plateau after the 4th pass with EUS-FNA, whereas it reached a plateau after the 2nd pass with EUS-FNB. EUS-FNB, endoscopic ultrasound-guided fine needle biopsy; EUSFNA, endoscopic ultrasound-guided fine needle aspiration.

value of cytology alone per session $(n=154)$ were $76 \%, 71 \%$, 100\%, 100\% and 40\%, respectively, while those of cytology alone per pass $(n=663)$ were 60\%, 52\%, 100\%, 100\% and 29\%. When the FNB and FNA groups were compared, accuracy per pass tended to be high in the FNB group: 64\% in the FNB group and $56 \%$ in the FNA group ( $\mathrm{p}=0.06$ ).

Blood contamination was more prominent in cytological specimen of the FNB group: blood contamination scores $\geq 2$ in $73 \%$ and 55\% of the FNB and FNA groups, respectively ( $<<0.01$ ). However, the cellularity score did not increase in the FNB group. The cellularity scores of $\geq 2$ were 59\% and 52\% in the FNB and FNA groups $(\mathrm{p}=0.07)$. 
Table 2. Prognostic Factors of First-Pass Tissue Acquisition

\begin{tabular}{|c|c|c|c|c|}
\hline \multirow{2}{*}{ Factor } & \multicolumn{2}{|c|}{ Univariate } & \multicolumn{2}{|c|}{ Multivariate } \\
\hline & OR $(95 \% \mathrm{CI})$ & p-value & OR $(95 \% \mathrm{CI})$ & p-value \\
\hline Age $\geq 70$ yr & $1.09(0.51-2.33)$ & 0.82 & - & - \\
\hline Male sex & $0.97(0.45-2.12)$ & 0.94 & - & - \\
\hline Pancreatic cancer & $1.75(0.79-3.90)$ & 0.17 & - & - \\
\hline Tumor size $\geq 20 \mathrm{~mm}$ & $1.81(0.82-3.99)$ & 0.14 & - & - \\
\hline Puncture site, stomach & $0.40(0.17-0.93)$ & 0.03 & $0.42(0.18-0.98)$ & 0.04 \\
\hline Needle type, FNB needle & $3.14(1.36-7.27)$ & 0.01 & $3.07(1.31-7.17)$ & 0.01 \\
\hline Endoscopist, expert & $1.16(0.54-2.48)$ & 0.70 & - & - \\
\hline
\end{tabular}

OR, odds ratio; CI, confidence interval; FNB, fine needle biopsy.

Table 3. The Diagnostic Performance of the Cytological Specimens

\begin{tabular}{|c|c|c|c|c|c|c|}
\hline \multirow{2}{*}{ Diagnostic performance } & \multicolumn{3}{|c|}{ Per session } & \multicolumn{3}{|c|}{ Per pass } \\
\hline & $\begin{array}{c}\text { FNB } \\
(\mathrm{n}=71)\end{array}$ & $\begin{array}{l}\text { FNA } \\
(\mathrm{n}=83)\end{array}$ & $\mathrm{p}$-value & $\begin{array}{c}\text { FNB } \\
(\mathrm{n}=300)\end{array}$ & $\begin{array}{c}\text { FNA } \\
(\mathrm{n}=363)\end{array}$ & $\mathrm{p}$-value \\
\hline Accuracy & 79 & 74 & 0.46 & 64 & 56 & 0.06 \\
\hline Sensitivity & 74 & 69 & 0.70 & 55 & 49 & 0.20 \\
\hline Specificity & 100 & 100 & 1.00 & 100 & 100 & 1.00 \\
\hline PPV & 100 & 100 & 1.00 & 100 & 100 & 1.00 \\
\hline NPV & 48 & 33 & 0.30 & 35 & 24 & 0.02 \\
\hline
\end{tabular}

Data are presented as percentage.

FNB, fine needle biopsy; FNA, fine needle aspiration; PPV, positive predictive value; NPV, negative predictive value.

Table 4. The Diagnostic Performance of EUS-FNB and EUS-FNA per Session, per Pass and at First Pass

\begin{tabular}{|c|c|c|c|c|c|c|c|c|c|}
\hline \multirow[b]{2}{*}{ Diagnostic performance } & \multicolumn{3}{|c|}{ Per session } & \multicolumn{3}{|c|}{ Per pass } & \multicolumn{3}{|c|}{ First pass } \\
\hline & $\begin{array}{l}\text { FNB } \\
(n=57)\end{array}$ & $\begin{array}{l}\text { FNA } \\
(\mathrm{n}=72)\end{array}$ & p-value & $\begin{array}{c}\text { FNB } \\
(n=241)\end{array}$ & $\begin{array}{c}\text { FNA } \\
(\mathrm{n}=312)\end{array}$ & $\mathrm{p}$-value & $\begin{array}{c}\text { FNB } \\
(\mathrm{n}=57)\end{array}$ & $\begin{array}{l}\text { FNA } \\
(n=72)\end{array}$ & p-value \\
\hline Histological tissue acquisition & 100 & 94 & 0.13 & 89 & 73 & $<0.01$ & 88 & 69 & 0.02 \\
\hline Histology positive for malignancy & 93 & 73 & $<0.01$ & 70 & 46 & $<0.01$ & 63 & 43 & 0.03 \\
\hline Cytology positive for malignancy & 74 & 69 & 0.70 & 55 & 49 & 0.20 & 47 & 46 & 1.00 \\
\hline Histology or cytology positive for malignancy & 97 & 85 & 0.04 & 79 & 64 & $<0.01$ & 72 & 58 & 0.14 \\
\hline
\end{tabular}

Data are presented as percentage.

EUS, endoscopic ultrasound; FNB, fine needle biopsy; FNA, fine needle aspiration.

\section{The diagnostic performance of EUS-FNB and EUS-FNA in malignancy}

A total of 553 passes were performed in 129 malignant cases and the diagnostic performance is shown in Table 4. Histological tissue acquisition rate per session was not significantly different: 100\% and 94\% in the FNB and FNA group ( $p=0.13$ ), respectively. However, sensitivity of histology alone per session was higher in the FNB group: 93\% and 73\% in the FNB and FNA group ( $\mathrm{p}<0.01$ ), respectively.

In the per pass analysis, EUS-FNB demonstrated a higher histological specimen acquisition rate $(89 \%$ and $73 \%, \mathrm{p}<0.01)$ and higher sensitivity in histology (70\% vs $46 \%, \mathrm{p}<0.01)$ per pass.
The sensitivity of cytology per pass was similar (55\% and 49\% in the FNB and FNA groups, $p=0.20$ ) and the combined histological and cytological sensitivity per pass was significantly higher (79\% and 64\% in the FNB and FNA groups, respectively, $\mathrm{p}<0.01)$. The incremental increase of the diagnostic performance by adding cytology was $4 \%$ and $14 \%$ in the FNB and FNA groups ( $\mathrm{p}=0.07$ ).

In the first-pass analysis, both the histological tissue acquisition rate ( $88 \%$ vs $69 \%, \mathrm{p}=0.02)$ and the histological sensitivity (63\% vs 43\%, p=0.03) were significantly higher in the EUS-FNB group, too. Prognostic factor analyses for histological sensitivity in a first pass are shown in Table 5. In the multivariate analysis, there were no statistically significant prognostic factors of first- 
Table 5. Prognostic Factors of First-Pass Histological Sensitivity

\begin{tabular}{|c|c|c|c|c|}
\hline \multirow{2}{*}{ Factor } & \multicolumn{2}{|c|}{ Univariate } & \multicolumn{2}{|c|}{ Multivariate } \\
\hline & OR $(95 \% \mathrm{CI})$ & $\mathrm{p}$-value & OR (95\% CI) & p-value \\
\hline Age $\geq 70$ yr & $0.90(0.44-1.84)$ & 0.76 & - & - \\
\hline Male sex & $1.01(0.49-2.11)$ & 0.97 & - & - \\
\hline Tumor size $\geq 20 \mathrm{~mm}$ & $2.23(0.99-5.05)$ & 0.05 & $2.15(0.95-4.91)$ & 0.07 \\
\hline Puncture site, stomach & $0.77(0.37-1.60)$ & 0.48 & - & - \\
\hline Needle type, FNB needle & $1.83(0.87-3.85)$ & 0.11 & $1.76(0.83-3.74)$ & 0.14 \\
\hline Endoscopist, expert & $0.86(0.43-1.72)$ & 0.67 & - & - \\
\hline
\end{tabular}

OR, odds ratio; CI, confidence interval; FNB, fine needle biopsy.

pass histological diagnostic sensitivity. However, EUS-FNB (OR, 1.76; $\mathrm{p}=0.14$ ) and the tumor size of $\geq 20 \mathrm{~mm}(\mathrm{OR}, 2.15 ; \mathrm{p}=0.07$ ) had a tendency toward positive malignancy in a first pass, though not statistically significant.

In the first-pass analysis of 111 pancreatic cancer cases (51 EUS-FNB and 60 EUS-FNA), the histological tissue acquisition rate was 96\% and 90\% ( $\mathrm{p}=0.28)$ and the diagnosis of definitive cancer was obtained in $84 \%$ and $63 \%$ by EUS-FNB and by EUSFNA ( $\mathrm{p}=0.02)$.

\section{DISCUSSION}

In our retrospective comparative analysis, EUS-FNB using a 22-gauge Franseen needle was associated with a higher tissue acquisition rate in the first pass compared with EUS-FNA using a 22-gauge standard needle in EUS-guided tissue acquisition of pancreatic solid lesions. While tissue acquisition rate reached a plateau after four passes of FNA, it reached a plateau after two passes of FNB, suggesting that this FNB needle would provide a higher acquisition rate of core tissue with fewer passes even without ROSE. The diagnostic performance of malignancy was also higher in the EUS-FNB group in per pass and per session analyses. The Franseen needle has three cutting edges to facilitate greater tissue acquisition and improve diagnostic ability. ${ }^{15}$ In our study, transgastric approach was associated with lower tissue acquisition rate in the first pass. In general, transduodenal puncture is technically difficult, especially when a 19-gauge or FNB needle is used. In a comparative study of 19- and 22-gauge FNA needles, ${ }^{25}$ while 22-gauge FNA needle demonstrated similar diagnostic accuracy throughout the pancreas, 19-gauge FNA needle was superior to 22-gauge FNA needle in the body and tail of pancreas, but not in the head of pancreas. In another study comparing 19-gauge FNA and reversed bevel FNB needles, ${ }^{9}$ FNB needle showed better diagnostic accuracy only by transgastric puncture. Given the better diagnostic performance in transduodenal puncture using this 22-Franseen needle, it is suggested that this needle has good technical feasibility even in the duodenum. In addition, the stroke technique might affect the study outcomes. In a comparative study of the stroke methods using 22-gauge FNA needle, ${ }^{26}$ the major study outcome is that the door-knocking method showed better diagnostic accuracy in the stomach. However, when the conventional stroke technique is used, the tissue acquisition rate was 100\% in the duodenum and $85.7 \%$ in the stomach. In our study cohort, the conventional stroke was used in most cases, rather than the door-knocking method, which might lead to the better tissue acquisition rate in the duodenum using the 22-gauge Franseen needle.

Although EUS-guided tissue acquisition has been established as a method for the pathological diagnosis of pancreatic lesions, there is some controversy on its techniques such as the needle size, the application of suction or a stylet, the number of passes and so on. EUS-FNB is a recent issue of debate in this field since a few types of FNB needles are now commercially available: Procore, ${ }^{4-9}$ fork-tip, ${ }^{11-14}$ and Franseen ${ }^{15-20}$ needles. There are comparative studies between FNB and FNA needles or between FNB needles with conflicting data but a recent meta-analysis of 11 randomized controlled trials demonstrated superiority of EUSFNB to EUS-FNA in terms of specimen adequacy, sensitivity and the number of passes. After the promising initial results of EUS-FNB using the Franseen needle, ${ }^{15}$ Bang et al. ${ }^{16}$ conducted a small randomized controlled trial comparing the Franseen needle and the conventional FNA needle, showing a larger total tissue, tumor and desmoplastic fibrosis were obtained by two dedicated passes for histological analysis by cell block. In another retrospective comparative study, the Franseen needle provided better tissue acquisition rate with a mean of 2.1 passes compared to 3.2 passes by the conventional FNA needle. ${ }^{19}$ Our study also confirmed similar findings with a larger cohort; histology acquisition rate of EUS-FNB reached a plateau after two passes with better sensitivity compared to EUS-FNA in per pass analysis.

While ROSE reportedly reduces the number of passes in EUS-FNA, ${ }^{27}$ it is not globally available in clinical practice. The optimal number of passes in EUS-FNA without ROSE is still unclear. ${ }^{28-30}$ While seven passes were required to obtain a sensitivity of $83.3 \%$ with a single pass sensitivity as low as $16.7 \%$ in a prospective study published in $2001,^{29}$ a recent prospective study demonstrated that sensitivity of $93 \%$ was reached within 
four passes if the pancreatic tumor was $>2 \mathrm{~cm}^{30}$ In our analysis, the number of passes necessary to reach a plateau of tissue acquisition was four in EUS-FNA and two in EUS-FNB without ROSE. A recent meta-analysis ${ }^{21}$ also showed that EUS-FNA and EUS-FNB were comparable in the presence of ROSE but that EUS-FNB was associated with a better diagnostic adequacy within fewer passes in pancreatic lesions. Macroscopic on-site quality evaluation ${ }^{31}$ was reportedly useful in EUS-FNA using a 19-gauge standard needle in the absence of ROSE but conflicting data were reported in EUS-FNA using a 22-gauge needle. ${ }^{32,33}$ Macroscopic on-site quality evaluation using 22-gauge Franseen needle showed promising results, with $>90 \%$ sensitivity. Given the relatively large histological core tissue obtained by the Franseen needle, specimen adequacy can be speculated by macroscopic observation.

There are some concerns on adverse events in EUS-FNB using the Franseen needle. While this Franseen needle can procure the tissue in the pancreatic lesion, three cutting edges can potentially injure the vessels in the gastrointestinal wall or the pancreas. In the initial report, ${ }^{15}$ one arterial bleeding (3\%), which required endoscopic hemostasis, was reported. In another study, ${ }^{34}$ two hematoma (4\%) and two post-procedure pain (4\%) were reported. In our study, there were no bleeding but two cases of pancreatitis (4\%) developed after EUS-FNB. We also noticed that temporary subclinical bleeding along the needle tract was prominent after EUS-FNB compared to EUS-FNA, similar to a previous report. ${ }^{19}$ These findings did not correlate with significant differences in the rate and severity of adverse events in our study but the adverse event rate appeared relatively high. Finally, it is important to clarify whether this bleeding may lead to the increase in tumor seeding or not in the long-term followup of a large study population.

Our study has some limitations. First, this was a single center, retrospective study with a relatively small study population. However, consecutive patients were included in the analysis and the needle selection was solely based on the chronological order, which we believe reduces the risk of selection bias. In addition, the availability of per pass analysis was the strength of our study. Second, our study design of historical cohorts can potentially lead to some bias. The results of EUS-guided tissue acquisition could be affected by the experiences of endoscopists and cytopathologists. In our study, EUS-FNB was more often performed by trainees and there were no significant differences in outcomes between experts and trainees. Therefore, we believe learning curve effects did not exist in our study. In addition, we have performed EUS-guided tissue acquisition for 20 years and the processing of procedure is standardized and it is unlikely that the cohort effect existed in the evaluation by cytopathologists in this study period. Third, the uniform criteria for quality and quantity in the histological specimen obtained by EUS-FNB were lacking. Fourth, we only included pancreatic solid lesions in our study. Although pancreatic solid lesions are the major target of EUS-guided tissue acquisition, the performance of the Franseen needle in evaluating lesions other than pancreatic masses should be further evaluated.

In conclusion, 22-gauge Franseen FNB needle was associated with a higher tissue acquisition rate in the first pass compared with 22-gauge standard FNA needle in EUS-guided tissue acquisition of pancreatic solid lesions.

\section{CONFLICTS OF INTEREST}

Y.N. and H.I. received a research grant from Boston Scientific Japan. The other authors declare that they have no conflicting interests.

\section{AUTHOR CONTRIBUTIONS}

Study concept and design: K.I., Y.N. Data acquisition: K.I., Y.N., H.O., S.K., T. Suzuki, T.N., T. Sato, R.H., K.S., T. Saito, N.T., T.H., S.M., H.K., M.T., H.I., K.K. Data analysis and interpretation: K.I., Y.N. Drafting of the manuscript; critical revision of the manuscript for important intellectual content: K.I., Y.N., H.I. Statistical analysis: K.I., Y.N. Obtained funding: Y.N., H.I. Administrative, technical, or material support; study supervision: Y.N., H.I.

\section{ORCID}

Kazunaga Ishigaki https://orcid.org/0000-0001-6773-9465 Yousuke Nakai https://orcid.org/0000-0001-7411-1385 Hiroki Oyama https://orcid.org/0000-0003-3591-821X Sachiko Kanai https://orcid.org/0000-0001-5856-6590 Tatsunori Suzuki https://orcid.org/0000-0002-2344-7732 Tomoka Nakamura https://orcid.org/0000-0002-0464-6269 Tatsuya Sato Ryunosuke Hakuta https://orcid.org/0000-0002-5142-4706 https://orcid.org/0000-0001-7653-7689 Kei Saito

Tomotaka Saito Naminatsu Takahara Tsuyoshi Hamada Suguru Mizuno Hirofumi Kogure Minoru Tada Hiroyuki Isayama Kazuhiko Koike https://orcid.org/0000-0003-2290-9373 https://orcid.org/0000-0001-6008-1648 https://orcid.org/0000-0002-1265-3100 https://orcid.org/0000-0002-3937-2755 https://orcid.org/0000-0001-7216-2269 https://orcid.org/0000-0002-2355-7309 https://orcid.org/0000-0001-5125-0964 https://orcid.org/0000-0002-3308-9326 https://orcid.org/0000-0002-9787-1907

\section{REFERENCES}

1. Hewitt MJ, McPhail MJ, Possamai L, Dhar A, Vlavianos P, Monahan KJ. EUS-guided FNA for diagnosis of solid pancreatic neoplasms: a meta-analysis. Gastrointest Endosc 2012;75:319331.

2. Puli SR, Bechtold ML, Buxbaum JL, Eloubeidi MA. How good is endoscopic ultrasound-guided fine-needle aspiration in diagnos- 
ing the correct etiology for a solid pancreatic mass? A metaanalysis and systematic review. Pancreas 2013;42:20-26.

3. Eltoum IA, Alston EA, Roberson J. Trends in pancreatic pathology practice before and after implementation of endoscopic ultrasound-guided fine-needle aspiration: an example of disruptive innovation effect? Arch Pathol Lab Med 2012;136:447-453.

4. Iglesias-Garcia J, Poley JW, Larghi A, et al. Feasibility and yield of a new EUS histology needle: results from a multicenter, pooled, cohort study. Gastrointest Endosc 2011;73:1189-1196.

5. Iwashita T, Nakai Y, Samarasena JB, et al. High single-pass diagnostic yield of a new 25-gauge core biopsy needle for EUSguided FNA biopsy in solid pancreatic lesions. Gastrointest Endosc 2013;77:909-915.

6. Larghi A, Iglesias-Garcia J, Poley JW, et al. Feasibility and yield of a novel 22-gauge histology EUS needle in patients with pancreatic masses: a multicenter prospective cohort study. Surg Endosc 2013;27:3733-3738.

7. Bang JY, Hawes R, Varadarajulu S. A meta-analysis comparing ProCore and standard fine-needle aspiration needles for endoscopic ultrasound-guided tissue acquisition. Endoscopy 2016;48:339-349.

8. Chong CCN, Teoh AYB, Tang RSY, Chan AWH, Ng EKW, Lai PBS. EUS-FNA using 22G nitinol or ProCore needles without on-site cytopathology. Endosc Ultrasound 2018;7:56-60.

9. Iwashita T, Nakai Y, Mukai T, et al. A 19-gauge histology needle versus a 19-gauge standard needle in endoscopic ultrasoundguided fine-needle aspiration for solid lesions: a multicenter randomized comparison study (GREATER Study). Dig Dis Sci 2018;63:1043-1051.

10. Fujie S, Ishiwatari H, Sasaki K, et al. Comparison of the diagnostic yield of the standard 22-gauge needle and the new 20-gauge forward-bevel core biopsy needle for endoscopic ultrasoundguided tissue acquisition from pancreatic lesions. Gut Liver 2019;13:349-355.

11. Kandel P, Tranesh G, Nassar A, et al. EUS-guided fine needle biopsy sampling using a novel fork-tip needle: a case-control study. Gastrointest Endosc 2016;84:1034-1039.

12. Jovani M, Abidi WM, Lee LS. Novel fork-tip needles versus standard needles for EUS-guided tissue acquisition from solid masses of the upper GI tract: a matched cohort study. Scand J Gastroenterol 2017;52:784-787.

13. Naveed M, Siddiqui AA, Kowalski TE, et al. A Multicenter comparative trial of a novel EUS-guided core biopsy needle (SharkCore $\left({ }^{\mathrm{TM}}\right)$ ) with the 22-gauge needle in patients with solid pancreatic mass lesions. Endosc Ultrasound 2018;7:34-40.

14. Attili F, Rimbaş M, Fantin A, et al. Performance of a new histology needle for EUS-guided fine needle biopsy: a retrospective multicenter study. Dig Liver Dis 2018;50:469-474.

15. Bang JY, Hebert-Magee S, Hasan MK, Navaneethan U, Hawes R, Varadarajulu S. Endoscopic ultrasonography-guided biopsy using a Franseen needle design: initial assessment. Dig Endosc 2017;29:338-346.
16. Bang JY, Hebert-Magee S, Navaneethan U, Hasan MK, Hawes $\mathrm{R}$, Varadarajulu S. EUS-guided fine needle biopsy of pancreatic masses can yield true histology. Gut 2018;67:2081-2084.

17. Mitri RD, Rimbaş M, Attili F, et al. Performance of a new needle for endoscopic ultrasound-guided fine-needle biopsy in patients with pancreatic solid lesions: a retrospective multicenter study. Endosc Ultrasound 2018;7:329-334.

18. Bang JY, Hebert-Magee S, Navaneethan U, Hasan MK, Hawes R, Varadarajulu S. Randomized trial comparing the Franseen and Fork-tip needles for EUS-guided fine-needle biopsy sampling of solid pancreatic mass lesions. Gastrointest Endosc 2018;87:14321438.

19. Mukai S, Itoi T, Yamaguchi $\mathrm{H}$, et al. A retrospective histological comparison of EUS-guided fine-needle biopsy using a novel franseen needle and a conventional end-cut type needle. Endosc Ultrasound 2019;8:50-57.

20. Ishikawa T, Kawashima H, Ohno E, et al. Clinical impact of EUSguided fine needle biopsy using a novel franseen needle for histological assessment of pancreatic diseases. Can J Gastroenterol Hepatol 2019;2019:8581743.

21. Khan MA, Grimm IS, Ali B, et al. A meta-analysis of endoscopic ultrasound-fine-needle aspiration compared to endoscopic ultrasound-fine-needle biopsy: diagnostic yield and the value of onsite cytopathological assessment. Endosc Int Open 2017;5:E363E375.

22. Nakai $\mathrm{Y}$, Isayama $\mathrm{H}$, Chang $\mathrm{KJ}$, et al. Slow pull versus suction in endoscopic ultrasound-guided fine-needle aspiration of pancreatic solid masses. Dig Dis Sci 2014;59:1578-1585.

23. Cotton PB, Eisen GM, Aabakken L, et al. A lexicon for endoscopic adverse events: report of an ASGE workshop. Gastrointest Endosc 2010;71:446-454.

24. Kanda Y. Investigation of the freely available easy-to-use software 'EZR' for medical statistics. Bone Marrow Transplant 2013;48:452-458.

25. Song TJ, Kim JH, Lee SS, et al. The prospective randomized, controlled trial of endoscopic ultrasound-guided fine-needle aspiration using 22G and 19G aspiration needles for solid pancreatic or peripancreatic masses. Am J Gastroenterol 2010;105:17391745.

26. Mukai S, Itoi T, Ashida R, et al. Multicenter, prospective, crossover trial comparing the door-knocking method with the conventional method for EUS-FNA of solid pancreatic masses (with videos). Gastrointest Endosc 2016;83:1210-1217.

27. Wani S, Mullady D, Early DS, et al. The clinical impact of immediate on-site cytopathology evaluation during endoscopic ultrasound-guided fine needle aspiration of pancreatic masses: a prospective multicenter randomized controlled trial. Am J Gastroenterol 2015;110:1429-1439.

28. Erickson RA, Sayage-Rabie L, Beissner RS. Factors predicting the number of EUS-guided fine-needle passes for diagnosis of pancreatic malignancies. Gastrointest Endosc 2000;51:184-190.

29. LeBlanc JK, Ciaccia D, Al-Assi MT, et al. Optimal number of 
EUS-guided fine needle passes needed to obtain a correct diagnosis. Gastrointest Endosc 2004;59:475-481.

30. Mohamadnejad M, Mullady D, Early DS, et al. Increasing number of passes beyond 4 does not increase sensitivity of detection of pancreatic malignancy by endoscopic ultrasound-guided fineneedle aspiration. Clin Gastroenterol Hepatol 2017;15:10711078.

31. Iwashita T, Yasuda I, Mukai T, et al. Macroscopic on-site quality evaluation of biopsy specimens to improve the diagnostic accuracy during EUS-guided FNA using a 19-gauge needle for solid lesions: a single-center prospective pilot study (MOSE study).
Gastrointest Endosc 2015;81:177-185.

32. Nguyen YP, Maple JT, Zhang Q, et al. Reliability of gross visual assessment of specimen adequacy during EUS-guided FNA of pancreatic masses. Gastrointest Endosc 2009;69:1264-1270.

33. Ishiwatari H, Sato J, Fujie $S$, et al. Gross visual inspection by endosonographers during endoscopic ultrasound-guided fine needle aspiration. Pancreatology 2019;19:191-195.

34. El Hajj II, Wu H, Reuss S, et al. Prospective assessment of the performance of a new fine needle biopsy device for EUS-guided sampling of solid lesions. Clin Endosc 2018;51:576-583. 